\title{
A systematic review of the influences of product placement in food stores on dietary behaviours and sales
}

\author{
Sarah Shaw ${ }^{1,2}$, Janis Baird $^{1,2}$ and Christina Vogel ${ }^{1,2}$ \\ ${ }^{1}$ MRC Lifecourse Epidemiology Unit, University of Southampton, Southampton, United Kingdom and \\ ${ }^{2}$ NIHR Southampton Biomedical Research Centre, University of Southampton and University Hospital Southampton \\ NHS Foundation Trust, Southampton, United Kingdom
}

\section{Abstract}

Introduction: Empirical evidence of the effects of product placement strategies on consumers' dietary and purchasing behaviours is limited. A systematic review was conducted to collate the evidence, both observational and intervention, about how food and beverage product placement (availability and positioning) in food stores, influence dietary behaviours and sales.

Materials and Methods: Nine databases were systematically searched, using both MeSH and free text terms, for articles published between 2005 and February 2019. Titles and abstracts were screened by one reviewer. If eligible, two reviewers performed data extraction and assessed each article for risk of bias in relation to the research question based on predefined criteria according to guidelines from the Centre for Reviews and Dissemination. Results were synthesised using a vote counting technique which recorded the significance level and the direction of the effect in relation to the expected relationship with health improvement. This systematic review was registered with Prospero CRD: 42016048826.

Results: The search yielded 16,342 potential articles of which 38, 17 observational and 21 intervention articles, met the inclusion criteria. The heterogeneous nature of these studies meant meta-analyses were not possible.

Two observational studies were classified as having a high risk of bias, 6 moderate and 9 as low risk of bias. Two intervention studies were classified as having moderate risk of bias and the remaining 19 as high risk of bias.

For observation studies, six studies included sales outcomes, nine included diet outcomes and four included BMI outcomes. For intervention studies, 19 included sales outcomes, four included diet outcomes and one included BMI outcomes. 76 outcomes from observational studies and 89 outcomes from intervention studies were included in the vote counting synthesis. $76 \%$ of observational outcomes showed associations with placement strategies in the expected direction for health improvement with $41 \%$ being significant. $72 \%$ of intervention outcomes showed associations in the expected direction with $33 \%$ being significant.

Discussion: The evidence suggests food and beverage placement strategies in food stores have the potential to support healthier food-related behaviours. The difficulties in conducting high-quality intervention studies may be a contributing factor to the large number of non-significant results that have been published. This review provides evidence in support of the UK Government's updated Childhood Obesity Plan to restrict the prominent placement of unhealthy food in food stores.

\section{Conflict of Interest}

"There is no conflict of interest". 